\title{
Non-linearly variable filters for spectro-imaging systems
}

Thomas Begou, Frédéric Lemarquis, Antonin Moreau, Fabien Lemarchand, Holger Reus, et al.

Thomas Begou, Frédéric Lemarquis, Antonin Moreau, Fabien Lemarchand, Holger Reus, Detlef Arhilger, Harro Hagedorn, Julien Lumeau, "Non-linearly variable filters for spectro-imaging systems," Proc. SPIE 11852, International Conference on Space Optics - ICSO 2020, 118521B (11 June 2021); doi: $10.1117 / 12.2599235$

SPIE Event: International Conference on Space Optics - ICSO 2021, 2021, Online Only 


\section{International Conference on Space Optics-ICSO 2020}

Virtual Conference

30 March-2 April 2021

Edited by Bruno Cugny, Zoran Sodnik, and Nikos Karafolas
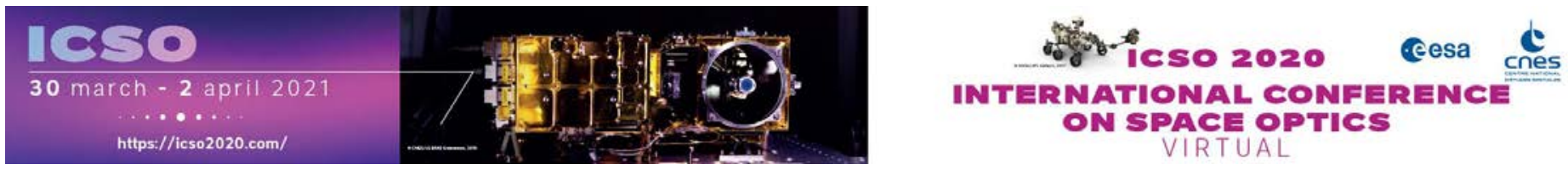

\section{Non-linearly variable filters for spectro-imaging systems}

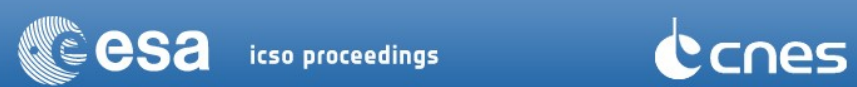




\title{
Non-linearly variable filters for spectro-imaging systems
}

\author{
Thomas Begou ${ }^{\mathrm{a},{ }^{*}}$, Frédéric Lemarquis ${ }^{\mathrm{a}}$, Antonin Moreau ${ }^{\mathrm{a}}$, Fabien Lemarchand $^{\mathrm{a}}$, Holger Reus ${ }^{\mathrm{b}}$, \\ Detlef Arhilger ${ }^{\mathrm{b}}$, Harro Hagedorn ${ }^{\mathrm{b}}$ and Julien Lumeau ${ }^{\mathrm{a}}$ \\ ${ }^{a}$ Aix Marseille Univ, CNRS, Centrale Marseille, Institut Fresnel, F-13013 Marseille, France \\ ${ }^{\mathrm{b}}$ Bühler Leybold Optics, Alzenau, Germany
}

\begin{abstract}
Variable filters, which central wavelength of the bandpass shifts along one dimension of the component, is a promising way to simplify and to miniaturize imaging spectrophotometer systems for spatial observation. In fact, by combining a dispersive element and a variable filter, it is possible to realize a spectral and a spatial filtering based on pushbroom technique. While there were numerous methods developed in order to produce variable filters, at this date, there is no simple, reliable and repeatable method. In this paper, we present a new method for the fabrication of variable filters based on plasma assisted reactive magnetron sputtering (PARMS).
\end{abstract}

Variable filters are obtained upon the variation of the thickness of some or all the layers deposited on the substrate. The layers are deposited using a Bühler HELIOS machine in order to benefit from the high stability of the deposition process. To insure a non-uniformity of the deposited layers, adapted masks are placed in front of the targets for the low and high refractive index materials. The uniformity of these layers is measured via a custom set-up allowing a local measurement of the transmission to perform a mapping of the transmission over the whole component aperture.

In this paper, we present the fabrication of layers and filters with variable thickness. Various types of thickness gradients are presented.

Keywords: Optical interferential filter, magnetron sputtering, variable filter

*thomas.begou@fresnel.fr

\section{INTRODUCTION}

Variable filters, which central wavelength of the bandpass shifts along one dimension of the component, is a promising way to simplify and to miniaturize imaging spectrophotometer systems for spatial observation. In fact, by combining a dispersive element and a variable filter, it is possible to realize a spectral and a spatial filtering based on pushbroom technique [1]. While there were numerous methods developed in order to produce variable filters, at this date, there is no simple, reliable and repeatable method. In this paper, we present a new method for the fabrication of variable filters based on plasma assisted reactive magnetron sputtering (PARMS).

Variable filters are obtained upon the variation of the thickness of some or all the layers deposited on the substrate. The layers are deposited using a Bühler HELIOS machine [2] in order to benefit from the high stability of the deposition process. To insure a non-uniformity of the deposited layers, adapted masks are placed in front of the targets for the low and high refractive index materials. The uniformity of these layers is measured via a custom set-up allowing a local measurement of the transmission to perform a mapping of the transmission over the whole component aperture [3].

We first present the results obtained for single layers of high and low refractive index materials, respectively $\mathrm{Nb}_{2} \mathrm{O}_{5}$ and $\mathrm{SiO}_{2}$. The results are used to validate the shape of the mask and to confirm the evolution of the thickness as a function of the position on the substrate.

We then demonstrate the fabrication of a narrow bandpass variable filter where the thicknesses of all the layers are changing, resulting in an overall shift of the filter. 


\section{EXPERIMENTAL SET UP TO CREATE A GRADIENT}

\subsection{Deposition system}

The deposition technology used in this work is plasma assisted reactive magnetron sputtering (PARMS) with a HELIOS machine from Bühler Optics. This set up, installed in the Espace Photonique of the Institut Fresnel, is constituted of:

$>12$ positions for substrate up to $100 \mathrm{~mm}$ diameter with one position dedicated to optical monitoring,

$>$ a rotating plate at 240 rounds per minute,

$>$ a load lock,

$>4$ processing zones (2 MF sputtering sources for dielectric material, $1 \mathrm{DC}$ sputtering source for metals and 1 assistance plasma),

$>\mathrm{a}$ in-situ optical monitoring in transmission,

$>$ available target: $\mathrm{Si}, \mathrm{Nb}, \mathrm{Hf}, \mathrm{Ta}, \mathrm{Ag}, \mathrm{Cr}$ and $\mathrm{Al}$,

$>$ available gas: $\mathrm{Ar}, \mathrm{O}_{2}$ and $\mathrm{N}_{2}$.

The HELIOS machine has been shown to be a very reliable machine for the deposition of very complex filters composed with up to several hundreds of layers. In particular, we have demonstrated complex bandpass filers within the framework of various space projects, e.g. IDEFIX, TARANIS or 3MI [4-6]. For these projects, deposition of multilayer stacks composed with more than one hundred layers with total thickness up to $25 \mu \mathrm{m}$ were needed and filters with spectral performances which do not deviate from theory by more than a few percent over broad spectral ranges were demonstrated. Within this project, we aim at fabricating a very narrowband filters with broad rejection for visible region. Preliminary designs have shown that $\sim 130$-layer stacks would be required for a total thickness $\sim 18 \mu \mathrm{m}$. The HELIOS machine, compared to classical plasma assisted evaporation set up, appears as highly appropriate for this application.

The work presented in this paper is the result of a close collaboration with Bülher Optics in Alzenau that aims at developing an adapted masking technology to generate a controlled thickness variation along one direction on the substrate.

\subsection{Masking technology}

The masking technology, developed for this application, relies on the control of the deposition time using the geometry of the deposition chamber. First, it is important to note that the deposition of material on a given substrate is intermittent since the substrate is on a rotating plate. The targets being fixed and positioned within a few millimeters from the substrates, the deposition occurs only when the substrate is under the targets, i.e. over an approximately $120 \times 120 \mathrm{~mm}^{2}$ zone. The deposited thickness is then proportional to the time during which the substrate is in front of the targets (i.e. the speed of rotation of the substrate holder) and the deposition rate of the material (function of the sputtering parameters). In nominal configuration, uniformity masks are placed between the turntable and the targets. These masks are commonly used to guarantee a very good uniformity of the deposited layers. The idea developed for this project consisted in generating new masks that will produce an opposite effect to those generally used and thus generate a thickness gradient.

The developed technique consists in keeping uniformity masks below the target which guarantee a uniform distribution of sputtered material and to add new masks directly on the shutter to control the deposition time of material along the radial direction on the substrate.

This type of shutter and mask were designed, made and then installed on the HELIOS machine of the Fresnel Institute, in collaboration with Bühler company. The control programs of the machine have also been adapted to allow the use of the mask.

Figure 1 shows a picture of the masking system, when the machine is open. When the machine is closed, the cover is positioned in front of the substrate holder and the shutter is then located a few millimeters from the substrate holder. In the left picture, the shutter is closed. This position makes it possible to completely hide the targets and thus to achieve target conditioning before the deposition. In the right picture, the same shutter is turned by $15^{\circ}$ in the so-called open position. The layers are deposited through this mask and thus it is possible to control the deposition time radially. 


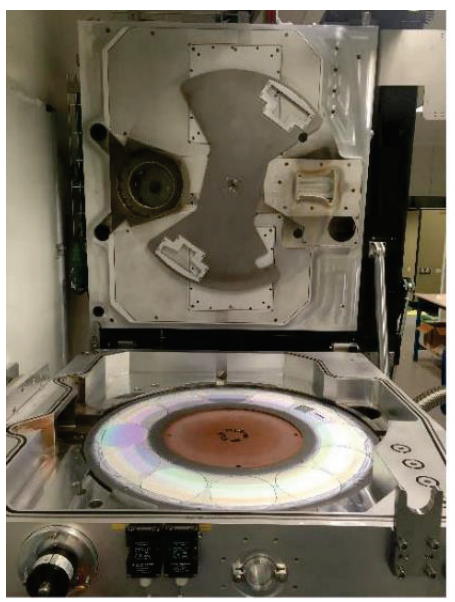

Shutter closed

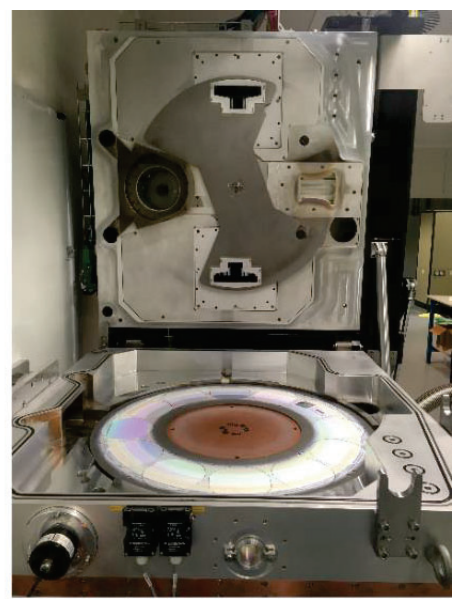

Shutter opened

Figure 1. Masking set up installed on the HELIOS deposition machine.

\subsection{Principle of the generation of a variable filter}

In order to validate the principle of a gradient generation, we designed a first mask, as illustrated in the Figure 2. It has 3 zones:

A uniform $35 \mathrm{~mm}$ area to perform in-situ optical monitoring. This area allows to control the thickness deposited in the region having the thinnest material thickness.

$>$ An area whose aperture varies by a factor of 3 over a distance of $10 \mathrm{~mm}$. It is this area that will be used to generate a thickness gradient with a ratio close to or equal to 3 .

$>$ Another uniform area in which aperture equal to $10 \mathrm{~mm}$. This area avoids the shading effects associated with the edge of the mask.
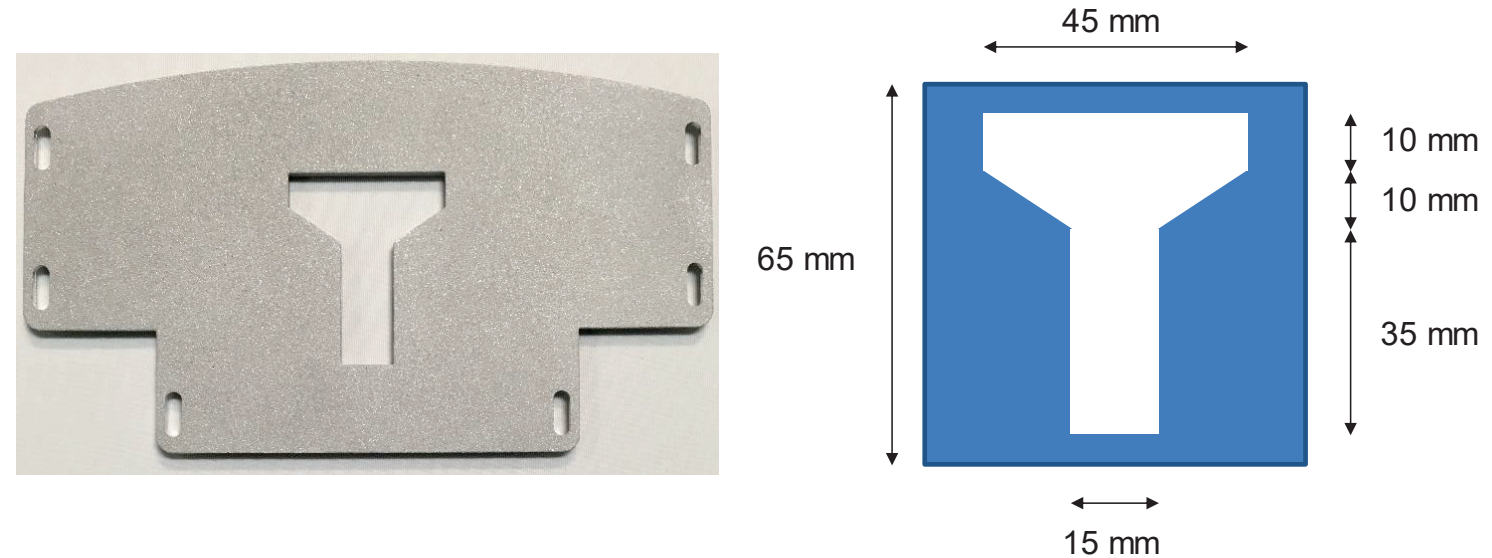

Figure 2. Photography and schematics of the first mask designed to generate variable filter.

A bandpass filter centered at $450 \mathrm{~nm}$ on the thinnest area has been deposited through the mask shown in Figure 2. The substrate on which the filter was deposited was then characterized in transmission using a uniformity bench installed within 
the Espace Photonique platform [3]. Figure 3 illustrates the evolution of the transmission due to the thickness gradient thus created along the $\boldsymbol{x}$ direction.

We can then identify different zones:

$>$ Between 0 and $5 \mathrm{~mm}$, the targets are completely masked and there is no deposition. The transmission is that of a bare substrate.

$>$ Between 5 and $10 \mathrm{~mm}$, this is the beginning of the $35 \mathrm{~mm}$ uniform area. A shadowing effects due to the edge of the mask are visible inducing the generation of a thickness gradient. This results in a redshift of the central wavelength of the filter.

$>$ Between 10 and $40 \mathrm{~mm}$, this is the uniform zone of the $35 \mathrm{~mm}$ area. We observe a quasi-uniform zone with a filter centered at $450 \mathrm{~nm}$.

$>$ Between 40 and $55 \mathrm{~mm}$, this is the area generating the gradient. We observe a shift of the central wavelength of the filter by a factor close to 3 allowing to shift from 450 to $1200 \mathrm{~nm}$.

$>$ Between 55 and $60 \mathrm{~mm}$, there is a fairly uniform area corresponding to a central wavelength of the filter equal to $1200 \mathrm{~nm}$.

$>$ Between 60 and $65 \mathrm{~mm}$, we observe the same effect as between 5 and $10 \mathrm{~mm}$ due to the edge of the mask. This produces a thickness gradient that results in a blue shift of the central wavelength of the filter.

$>$ Between 65 and $70 \mathrm{~mm}$, the targets are completely masked again and there is no deposition. The transmission is that of a bare substrate.

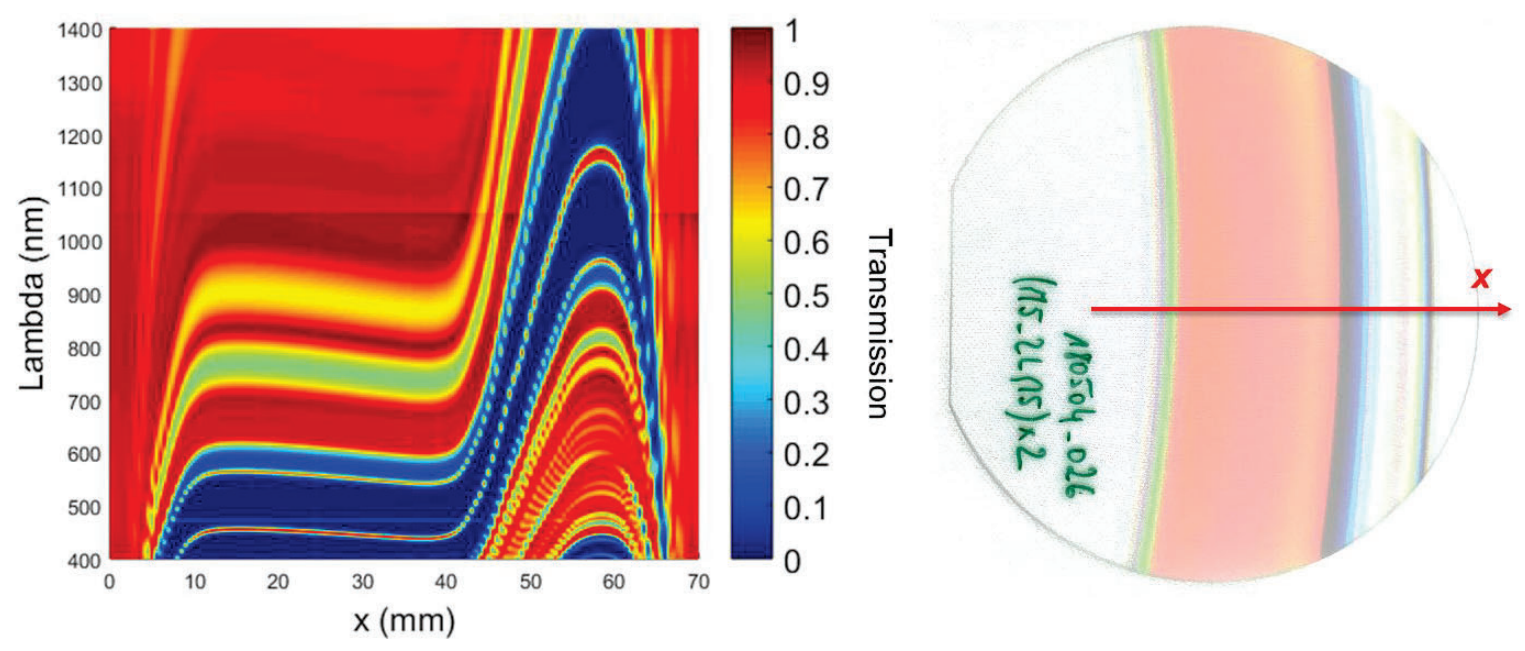

Figure 3. Evolution of the transmission of a variable filter deposited through the mask shown in Figure 2 as a function of the $\boldsymbol{x}$ direction.

Figure 3 validates the feasibility of a linear gradient filter with this method. The variation of the central wavelength is close to 3 over a distance of $10 \mathrm{~mm}$. It should therefore be noted that the deposition rate, with this mask, is reduced by a factor between 3 and 4 for the thickest zone. This means that the $18 \mu \mathrm{m}$ final filter that we aim would require a deposition time greater than 50 hours. However, this method allows further increase of the aperture of the gradient masks that would allow depositing at rates no lower than half of regular deposition rates. Moreover, as seen on the right part in Figure 3, according to the method used to generate the gradient, a curvature of the iso-thickness lines will occur. The expected "smile" should be around $15 \mu \mathrm{m}$ over a $5 \mathrm{~mm}$ filter width and should reach $50 \mu \mathrm{m}$ over a $10 \mathrm{~mm}$ filter width. This value will be characterized later but should not be a problem since the filter is used in front of a camera that will numerically correct this defect. 


\section{DEPOSITION OF SINGLE LAYER WITH VARIABLE THICKNESS}

In order to precisely characterize the generated gradients, we have carried out the deposition of two simple structures:

$>$ a single layer of $\mathrm{Nb}_{2} \mathrm{O}_{5}$ deposited through an adapted mask (see 2.3 part) on a silica substrate,

$>$ a single layer of $\mathrm{SiO}_{2}$ deposited through the same mask on a silica substrate previously coated with a uniform $\mathrm{Nb}_{2} \mathrm{O}_{5}$ layer.

The mask, used to perform the gradient, has a structure very similar to that shown in Figure 2. Contrary to the mask presented in the above section, an unmasked area of $5 \mathrm{~mm}$ appears between the uniform and the gradient zones. The shape ratio between the thinner and the thicker part is of 2 . The local transmission is measured with the uniformity measuring bench and then, by reverse engineering, the local thickness of the deposited layer is calculated (Figure 4).

We can see on each graph in Figure 4 that 2 zones are present:

$>$ an area between 9 and $19 \mathrm{~mm}$ with a thickness variation by a factor close to 2 (165 to $300 \mathrm{~nm}$ ),

$>$ a uniform zone between 30 and $45 \mathrm{~mm}$ with a thickness equal to $165 \mathrm{~nm}$.

It can also be noted that the gradient is almost linear, and that the gradients generated by the $\mathrm{Nb}_{2} \mathrm{O}_{5}$ or $\mathrm{SiO}_{2}$ targets are very close, which should make it possible the production of high performance variable filters.
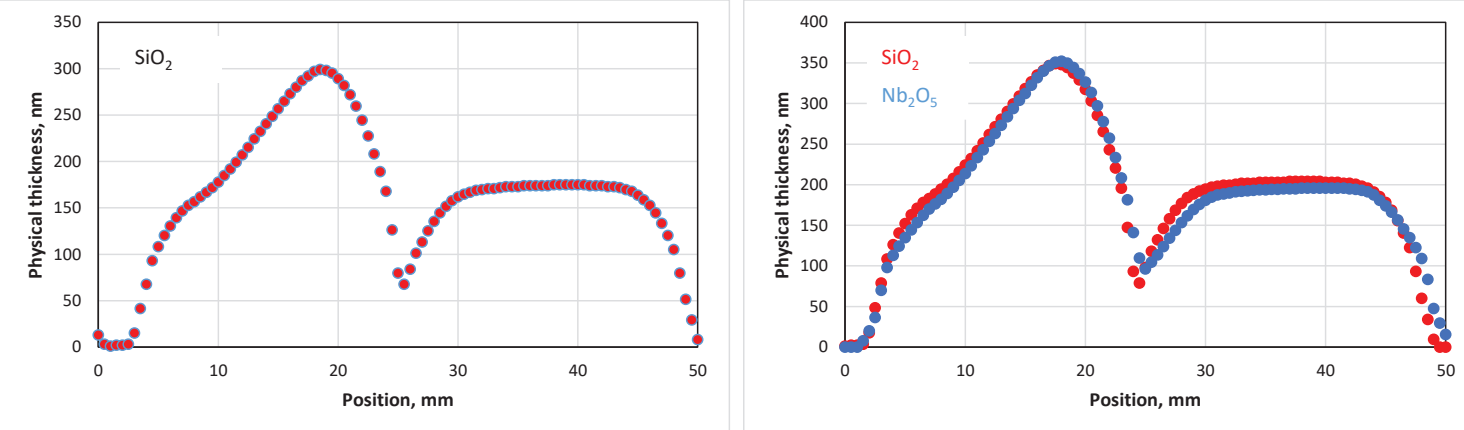

Figure 4. Gradient characterization for single layers of $\mathrm{SiO}_{2}$ and $\mathrm{Nb}_{2} \mathrm{O}_{5}$.

\section{DEPOSITION OF A NARROW BANDPASS FILTER WITH VARIABLE THICKNESS}

In order to experimentally validate our ability to achieve a narrow bandpass filter similar to that required for this project, we have tested the fabrication of a very narrow bandpass filter (without blocking filters) composed of a double FabryPerot cavity with 23 layers with the following structure:

\section{$\left[(H \mathrm{~L})^{2} \mathrm{H} 2 \mathrm{~L} \mathrm{H}(\mathrm{L} \mathrm{H})^{2}\right]$ L [(H L $)^{2}$ H $\left.2 \mathrm{~L} \mathrm{H}(\mathrm{L} \mathrm{H})^{2}\right]$}

where $\mathbf{H}$ and $\mathbf{L}$ are quarter-wave optical thickness layers at a $450 \mathrm{~nm}$ wavelength respectively made of $\mathrm{Nb}_{2} \mathrm{O}_{5} \mathrm{and}_{\mathrm{SiO}_{2}}$. The filter was deposited using the HELIOS machine with the uniformity masks of Figure 2 positioned below each target. The evolution of the spectral response of the filter along the $\boldsymbol{x}$ direction (as shown in Figure 3 ) has been measured with the uniformity bench and is represented in Figure 5. Figure 6 illustrates the experimental and theoretical transmission for 2 different $\boldsymbol{x}$ positions.

Figures 5 and 6 validate the fact that we have been able to produce a very narrow band linearly variable filter with performances close to theoretical performance. This shows that, provided that blocking filters are added, this masking method appears as an appropriate method to produce linearly and non-linearly variable filters. 

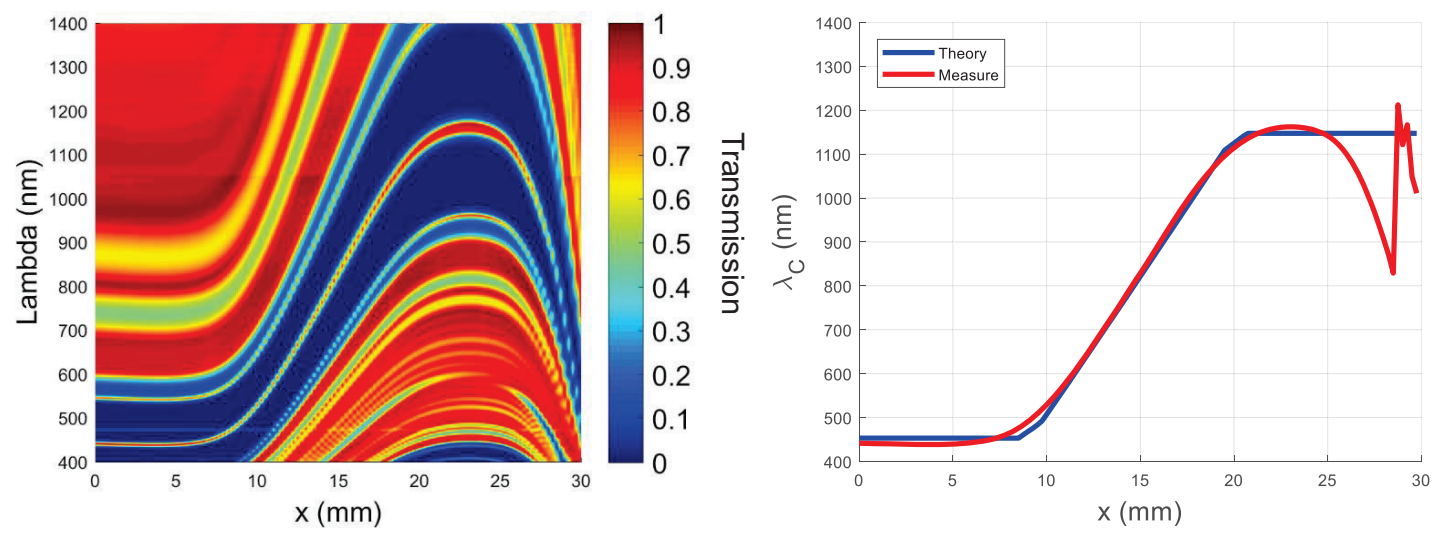

Figure 5. Evolution of the transmission of the narrow bandpass filter (on the left) and of the theoretical and experimental centering wavelength of the same filter (on the right).
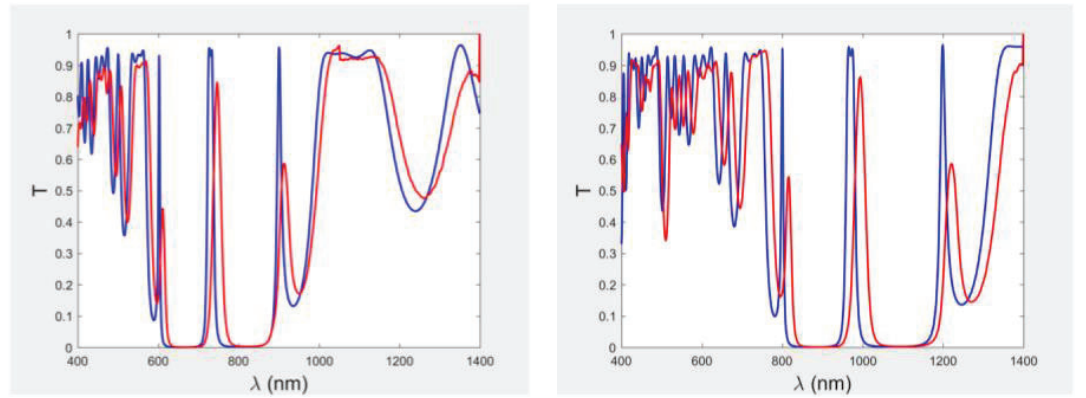

Figure 6. Evolution of the theoretical (blue) and experimental (red) transmission of the narrow bandpass filter for two different $\boldsymbol{x}$ positions.

\section{CONCLUSION}

We have demonstrated in this paper an efficient method for the generation of variable thickness filters and compatible with a Bühler HELIOS deposition machine and allowing the fabrication of complex filters. This method relies on the use of two uniformity masks, one for each material, fixed on the shutter. We have then demonstrated that this method could be used for the fabrication of narrowband filters and a first experimental demonstration was presented.

\section{REFERENCES}

[1] P. Mouroulis, R. O. Green, and T. G. Chrien, "Design of pushbroom imaging spectrometers for optimum recovery of spectroscopic and spatial information", Appl. Opt., vol. 39 (13), pp. 2210-2220, May 2000.

[2] M. Scherer, J. Pistner, W. Lehnert, "UV- and VIS Filter Coatings by Plasma Assisted Reactive Magnetron Sputtering (PARMS)", OIC 2010 Proceedings.

[3] L. Abel-Tiberini, F. Lemarquis and M. Lequime, "Dedicated spectrophotometer for localized transmittance and reflectance measurements," Appl. Opt., vol.45 (7), pp. 1386-1391, March 2006.

[4] T. Begou, H. Krol, D. Stojcevski, F. Lemarchand, M. Lequime, et al.. "Complex optical interference filters with stress compensation for space applications”. CEAS Space Journal, 2017. 
[5] T. Begou, H. Krol, C. Hecquet, C. Bondet, J. Lumeau, C. Grezes-Besset, and M. Lequime, "Optical filters for UV to near IR space applications", International Conference on Space Optics (Tenerife, Spain), October 2014.

[6] R. Le Goff, H. Krol, C. Grèzes-Besset, M. Lequime, T. Begou, C. Hecquet, M. Zerrad, B. Badoil, G. Montay, K. Gasc, "Multispectral filters assemblies for Earth remote sensing imagers", International Conference on Space Optics (Tenerife, Spain), October 2014. 